\title{
An Analysis of Two Genome-wide Association Meta-analyses Identifies a New Locus for Broad Depression Phenotype
}

\section{Direk, Nese}

2017-09-01

Direk, N , Williams , S , Smith , J A, Ripke , S, Air , T, Amare , A T, Amin , N, Baune , B T , Bennett, D A, Blackwood, D H R, Boomsma, D, Breen, G, Buttenschon, H N, Byrne , E M , Borglum , A D , Castelao , E, Cichon, S, Clarke , T-K, Cornelis, M C, Dannlowski , U , De Jager , P L , Demirkan , A , Domenici , E , van Duijn , C M , Dunn , E C , Eriksson , J G, Esko, T, Faul , J D , Ferrucci , L, Fornage , M , de Geus , E, Gill , M , Gordon , S D , Grabe , H J , van Grootheest, G, Hamilton , S P , Hartman , C A , Heath , A C , Hek , K, Hofman , A , Homuth , G , Horn , C , Hottenga , J J , Kardia , S L R , Kloiber , S, Koenen , K , Kutalik, Z, Ladwig , K-H , Lahti , J, Levinson, D F , Lewis , C M , Lewis , G , Li , Q S , Llewellyn, D J, Lucae , S , Lunetta , K L , Maclntyre , D J , Madden , P , Martin , N G , McIntosh , A M , Metspalu , A , Milaneschi , Y, Montgomery , G W , Mors , O, Mosley , T H , Murabito , J M , Mueller-Myhsok, B , Nothen, M M , Nyholt, D R, O'Donovan, M C , Penninx , B W , Pergadia , M L , Perlis , R , Potash , J B , Preisig , M , Purcell , S M , Quiroz , J A , Raikkonen, K, Rice, J P, Rietschel , M , Rivera, M , Schulze , T G, Shi , J , Shyn , S , Sinnamon, G C , Smit, J H , Smoller, J W , Snieder, H, Tanaka, T , Tansey , K E , Teumer, A, Uher , R, Umbricht, D, Van der Auwera, S, Ware , E B , Weir , D R , Weissman, M M , Willemsen, G , Yang , J , Zhao , W , Tiemeier , H \& Sullivan , P F 2017 , ' An Analysis of Two Genome-wide Association Meta-analyses Identifies a New Locus for Broad Depression Phenotype ' , Biological Psychiatry , vol. 82 , no. 5 , pp. 322-329 . https://doi.org/10.1016/j.biopsyc

http://hdl.handle.net/10138/297903

https://doi.org/10.1016/j.biopsych.2016.11.013

unspecified

publishedVersion

Downloaded from Helda, University of Helsinki institutional repository.

This is an electronic reprint of the original article.

This reprint may differ from the original in pagination and typographic detail.

Please cite the original version. 


\section{An Analysis of Two Genome-wide Association Meta-analyses Identifies a New Locus for Broad Depression Phenotype}

Nese Direk, Stephanie Williams, Jennifer A. Smith, Stephan Ripke, Tracy Air, Azmeraw T. Amare, Najaf Amin, Bernhard T. Baune, David A. Bennett, Douglas H.R. Blackwood, Dorret Boomsma, Gerome Breen, Henriette N. Buttenschøn, Enda M. Byrne, Anders D. Børglum, Enrique Castelao, Sven Cichon, Toni-Kim Clarke, Marilyn C. Cornelis, Udo Dannlowski, Philip L. De Jager, Ayse Demirkan, Enrico Domenici, Cornelia M. van Duijn, Erin C. Dunn, Johan G. Eriksson, Tonu Esko, Jessica D. Faul, Luigi Ferrucci, Myriam Fornage, Eco de Geus, Michael Gill, Scott D. Gordon, Hans Jörgen Grabe, Gerard van Grootheest, Steven P. Hamilton, Catharina A. Hartman, Andrew C. Heath, Karin Hek, Albert Hofman, Georg Homuth, Carsten Horn, Jouke Jan Hottenga, Sharon L.R. Kardia, Stefan Kloiber, Karestan Koenen, Zoltán Kutalik, Karl-Heinz Ladwig, Jari Lahti, Douglas F. Levinson, Cathryn M. Lewis, Glyn Lewis, Qingqin S. Li, David J. Llewellyn, Susanne Lucae, Kathryn L. Lunetta, Donald J. Maclntyre, Pamela Madden, Nicholas G. Martin, Andrew M. Mclntosh, Andres Metspalu, Yuri Milaneschi, Grant W. Montgomery, Ole Mors, Thomas H. Mosley Jr., Joanne M. Murabito, Bertram Müller-Myhsok, Markus M. Nöthen, Dale R. Nyholt, Michael C. O’Donovan, Brenda W. Penninx, Michele L. Pergadia, Roy Perlis, James B. Potash, Martin Preisig, Shaun M. Purcell, Jorge A. Quiroz, Katri Räikkönen, John P. Rice, Marcella Rietschel, Margarita Rivera, Thomas G. Schulze, Jianxin Shi, Stanley Shyn, Grant C. Sinnamon, Johannes H. Smit, Jordan W. Smoller, Harold Snieder, Toshiko Tanaka, Katherine E. Tansey, Alexander Teumer, Rudolf Uher, Daniel Umbricht, Sandra Van der Auwera, Erin B. Ware, David R. Weir, Myrna M. Weissman, Gonneke Willemsen, Jingyun Yang, Wei Zhao, Henning Tiemeier, and Patrick F. Sullivan

\footnotetext{
ABSTRACT

BACKGROUND: The genetics of depression has been explored in genome-wide association studies that focused on either major depressive disorder or depressive symptoms with mostly negative findings. A broad depression phenotype including both phenotypes has not been tested previously using a genome-wide association approach. We aimed to identify genetic polymorphisms significantly associated with a broad phenotype from depressive symptoms to major depressive disorder.

METHODS: We analyzed two prior studies of 70,017 participants of European ancestry from general and clinical populations in the discovery stage. We performed a replication meta-analysis of 28,328 participants. Single nucleotide polymorphism (SNP)-based heritability and genetic correlations were calculated using linkage disequilibrium score regression. Discovery and replication analyses were performed using a $p$-value-based meta-analysis. Lifetime major depressive disorder and depressive symptom scores were used as the outcome measures.

RESULTS: The SNP-based heritability of major depressive disorder was 0.21 (SE $=0.02$ ), the SNP-based heritability of depressive symptoms was 0.04 (SE $=0.01$ ), and their genetic correlation was 1.001 (SE $=0.2$ ). We found one genome-wide significant locus related to the broad depression phenotype (rs9825823, chromosome 3: 61,082,153, $p=8.2 \times 10^{-9}$ ) located in an intron of the FHIT gene. We replicated this SNP in independent samples $(p=.02)$ and the overall meta-analysis of the discovery and replication cohorts $\left(1.0 \times 10^{-9}\right)$.

CONCLUSIONS: This large study identified a new locus for depression. Our results support a continuum between depressive symptoms and major depressive disorder. A phenotypically more inclusive approach may help to achieve the large sample sizes needed to detect susceptibility loci for depression.
} 
Keywords: CHARGE consortium, Depressive symptoms, FHIT gene, Genome-wide association study, Major depressive disorder, Psychiatric Genomics Consortium

http://dx.doi.org/10.1016/j.biopsych.2016.11.013

The etiology of depression-a worldwide leading cause of disability (1)-is not well understood. As indicated by family, twin, and adoption studies, genetic factors mediate part of vulnerability to major depressive disorder (MDD) with a modest heritability of around $40 \%$ (2). However, we understand little of the specific genetic architecture of MDD. Multiple genomewide association studies (GWASs) for MDD have been published (3-10). The largest MDD GWAS was the mega-analysis by the MDD Working Group of the Psychiatric Genomics Consortium (PGC). In that study, more than 9000 MDD cases and 9500 control subjects were analyzed, but no association with MDD reached genome-wide significance (7). Recently, the CONVERGE (China, Oxford, and VCU Experimental Research on Genetic Epidemiology) consortium identified two genomewide significant associations in 5303 Chinese women with severe and recurrent MDD (near the SIRT1 gene, $p=2.53 \times$ $10^{-10}$, and in an intron of the LHPP gene, $p=6.45 \times 10^{-12}$ ) (11). A GWAS of depressive symptoms (23\%-29\% heritability) $(12,13)$ in the Cohorts for Heart and Aging Research in Genomic Epidemiology (CHARGE) consortium in approximately 50,000 people from the general population found no genome-wide significant associations (14). Owing to the relatively small sample sizes, the previous GWASs of depressive disorders and depressive symptoms were arguably underpowered to detect small genetic effects $(15,16)$.

Depression can be conceptualized along a continuum of severity from subthreshold or minor depression to MDD of varying severity (e.g., mild, moderate, severe) (17). Using a continuum approach may augment statistical power because sample size can be increased substantially and patients who fall into the gray area can be assessed. Several lines of evidence support a depression continuum. In longitudinal studies, there is an increased risk of MDD in patients with minor depression and subthreshold depression $(18,19)$. Statistical studies of disorder classification (taxometric) suggested that severity of depression is continuously distributed and that there is no discontinuity in the latent structure of depression $(19,20)$. Family studies report that relatives of probands with milder forms of depression have greater risk of MDD compared with relatives of probands without any mood disorders (21-24). A higher number of depressive symptoms is related to greater disability, worse quality of life, and higher mortality risk (18,25-29). MDD and continuous measures of depression are highly correlated, and severity of depressive symptoms along the continuum is linear $(30,31)$.

The goal of the current study was to combine the results of the largest GWAS using categorical lifetime MDD and continuous measures of depression to identify genetic variants underlying the entire depression continuum.

\section{METHODS AND MATERIALS}

\section{Study Design and Samples}

This study was a collaboration between investigators on the PGC MDD and CHARGE genome-wide association metaanalyses (GWAMA). In the discovery phase, we aggregated two GWAMAs published in 2013 (7,14). Basic descriptive features and phenotype definitions of the contributing samples are provided in Supplemental Table S1. The mega-analysis of MDD consisted of nine studies of 9240 cases meeting international criteria for lifetime MDD and 9519 healthy control subjects. The CHARGE meta-analysis of depressive symptoms included 22 cohorts and comprised 51,258 persons. Each cohort contributing to the GWAMA of the PGC and CHARGE was distinct. In the replication analyses, 16 casecontrol studies with DSM-IV MDD (6718 cases and 13,453 control subjects) were included along with 8157 subjects from the general population with assessment of depressive symptoms. All subjects were of European ancestry. Institutional review boards approved all studies, and all participants provided written informed consent.

\section{Phenotype Characteristics}

In the PGC GWAMA, MDD was established with structured clinical interviews (e.g., Clinical Interview Schedule-Revised, Diagnostic Interview for Genetic Studies, and Structured Clinical Interview for DSM-IV). All clinical evaluations were made by experienced clinicians or interviewers. Most cases were ascertained from clinical sources. Control subjects were screened in most of the studies to require the absence of MDD and were recruited from the general population. Full details about the PGC samples can be found in the previous publication (7). In the CHARGE GWAMA, depressive symptoms were assessed with validated questionnaires. Measures include the Center for Epidemiological Studies-Depression scale, Geriatric Depression Scale, Patient Health Questionnaire-9, and Beck Depression Inventory-II, mostly assessing depressive symptoms during previous weeks rather than lifetime MDD (14). Persons with schizophrenia, bipolar disorder, or dementia were excluded. Persons aged 40 years or older with genotype data and depressive symptom scores were included.

The 16 MDD case-control replication samples were part of an expanded but unpublished PGC MDD analysis. MDD was diagnosed with interviews. In the depressive symptom replication cohort, the Health and Retirement Study, the 8-item Center for Epidemiological Studies-Depression scale was applied. Respondents were excluded if they were under 40 years of age or displayed evidence of cognitive impairment. 


\section{Genotyping and Imputation}

In the PGC samples (Supplemental Table S1), individual genotypes were assembled, processed through a central quality control pipeline, and imputed using the CEU (Central Europe) and TSI (Toscani in Italy) HapMap3 reference panels. Quality control procedures were extensive (7). In the CHARGE cohorts, genotype quality control and imputation were conducted in each study separately. The imputation reference was the HapMap2 CEU panel (14). In the MDD replication cohorts (Supplemental Table S3), imputation was performed using IMPUTE2 or SHAPEIT (chunk size of $3 \mathrm{Mb}$ and default parameters). The imputation reference set consisted of 2186 phased haplotypes from the 1000 Genomes Project. In the Health and Retirement Study, imputation was performed using the HapMap2 CEU reference panel.

\section{Statistical Analyses}

Linkage disequilibrium score regression was used to compute the single nucleotide polymorphism (SNP)-based heritability and the genetic correlation using the 1000 Genomes CEU reference panel (32).

In the PGC GWAMA, a logistic regression analysis was used to test the association between MDD and imputed SNP dosages under an additive model and adjusting for study indicators and five principal components (7). In the CHARGE GWAMA, a linear regression analysis was applied to test the association of depressive symptom score on imputed SNP dosages in the contributing studies adjusting for age and sex. Analyses were adjusted for principal components for most, but not all, cohorts in the CHARGE GWAMA. A $p$-value-based meta-analysis was applied in the CHARGE GWAMA (14). Effect size estimates were based on a dichotomous outcome in the PGC and on a continuous outcome in the CHARGE GWAMA. To combine these effect estimates, a $p$-value-based meta-analysis weighted by sample size with METAL (http://www.sph.umich.edu/csg/ abecasis/metal/) was used. This method allows different weights for each study and takes into account the direction of effect at each SNP (33). To specify the direction of the effect, the PGC used the logistic regression coefficient beta and the CHARGE used $z$ scores. Weights were based on the number of MDD cases in the PGC study ( $n=9240)$, and the number of individuals in the CHARGE with clinically significant depressive symptoms ( $n=5976$ ) using population-specific cutoff scores of the questionnaires was considered for weighting. To test whether the results are affected by different sample size weightings, equal weights per study, or no weight as suggested by Stouffer et al. (34), we carried out a series of sensitivity analyses.

We selected the genome-wide significant SNPs in two loci from the discovery stage for replication. After analyzing these data, we performed a $p$-value-based meta-analysis combining all replication samples. Furthermore, we analyzed the results of the discovery and all replication samples weighting for number of cases.

\section{RESULTS}

In the discovery stage, we performed a GWAMA in 70,017 participants of European ancestry by combining the PGC MDD (7) and CHARGE GWAMA (14). We applied a linkage disequilibrium score regression to the summary statistics from each study to compute the SNP-based heritabilities and the genetic correlation. As reported previously (35), the SNPbased liability scale heritability of MDD was $0.2(\mathrm{SE}=0.02)$ for $20 \%$ of prevalence. The lambda was 1.1 and the regression intercept was 1.0 (SE $=0.01)$. The SNP-based heritability of depressive symptoms was 0.04 (SE $=0.01$ ). The lambda was 1.1 and the regression intercept was 1.0 (SE $=0.01)$. The SNP-based heritability of the broad depression phenotype was $0.3(\mathrm{SE}=0.04)$. MDD and depressive symptoms showed significant coheritability $(1.001, \mathrm{SE}=0.2, z$ score $=4.6, p=$ $\left.4.6 \times 10^{-6}\right)$. This result supports the contention of a continuum between depressive symptoms and MDD. However, the genetic correlation should be interpreted carefully because linkage disequilibrium regression is quite sensitive to environmental confounding and, like twin studies, often lacks precision. In addition, different evaluation methods of the depression phenotypes might cause different genetic correlation estimates that cannot easily be compared.

We conducted a meta-analysis of the PGC MDD and the CHARGE depressive symptoms GWAMA using a weighted, $p$-value-based meta-analysis. The results are summarized in Figure 1 and Supplemental Figures S1 to S3. The combined meta-analysis was conducted for 918,921 SNPs. Two loci were genome-wide significant: an SNP in an intron of the FHIT gene (rs9825823, chromosome 3: 61,082,153, $p=8.2 \times 10^{-9}$ ) and an SNP in an intron of PLEK2 (rs9323497, chr14: $67,873,128, p=3.3 \times 10^{-8}$ ) (Table 1). All SNPs with a $p$ value of association $<5 \times 10^{-5}$ are presented in Supplemental Table S2. Using different weights or Stouffer's unweighted method had only slight effects on the results (data not shown). Supplemental Figures S4 and S5 show forest plots for two SNPs shown in Table 1.

Table 2 presents the replication analyses and the metaanalysis of discovery and replication results. One of the genome-wide significant variants within the FHIT gene (rs9825823) was associated with the depression continuum in the replication cohorts ( $z$ score $=2.4, p=.02$ ). The result of the final meta-analysis of discovery and replication samples also indicated a positive replication as indexed by a lower $p$ value $\left(z\right.$ score $\left.=6.1, p=1.0 \times 10^{-9}\right)$. This SNP had a positive association with depressive symptoms in the CHARGE study $\left(p=5.5 \times 10^{-4}\right)$, and a similar pattern was observed in the PGC study $\left(p=4.1 \times 10^{-6}\right)$. The SNP in an intron of PLEK2 (rs9323497) was not related to the depression continuum significantly ( $z$ score $=0.2, p=.90$ ).

We performed an additional replication analysis of our two genome-wide significant SNPs using the publicly available data of the recently published GWAMA of depressive disorders in a sample of Chinese women (the CONVERGE study) (11). In CONVERGE, rs9825823 (odds ratio $=1.01, p=.12$ ) and rs9323497 (odds ratio $=0.97, p=.0002$, with a different direction of association than in our discovery sample) were not related to depression at the genome-wide significance level, although the latter reached nominal significance. However, in the joint meta-analysis of the Health and Retirement Study, the PGC MDD study, and CONVERGE study, we found that the association between rs9825823 and the depression continuum ( $z$ score $=2.85, p=.004$ ) was slightly stronger than our initial replication analysis. When these replication and 


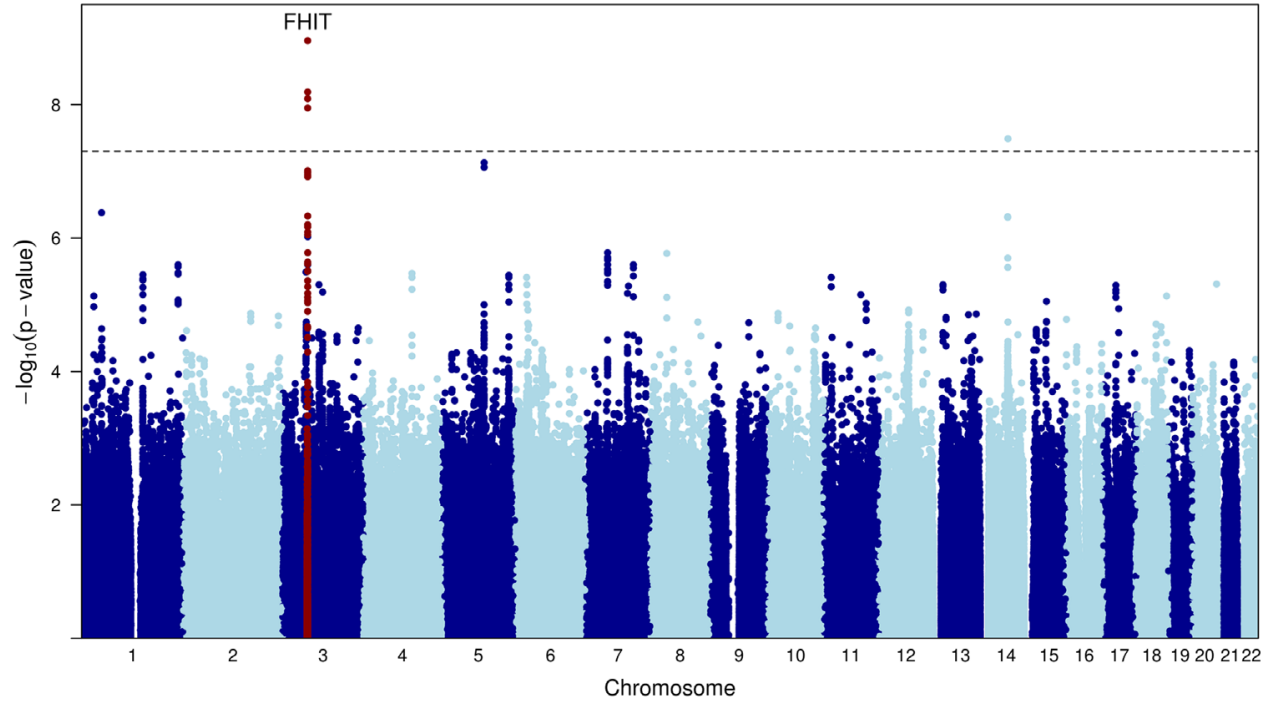

Figure 1. Manhattan plot. The $x$ axis represents the chromosomal position for each SNP, and the $y$ axis represents the $-\log _{10} p$ value for association with depression. discovery samples were combined, the association with our top hit also became stronger (analysis without the CONVERGE data: $z$ score $=6.1, p=1 \times 10^{-9}$; analysis with the CONVERGE data: $z$ score $\left.=6.2, p=6.8 \times 10^{-10}\right)$. Results of additional replication analyses are given in Supplemental Table S4.

\section{DISCUSSION}

We report the results of a combined GWAMA of the depression continuum including MDD (18,759 cases and control subjects) and depressive symptoms (51,258 participants). In the discovery stage, we found genome-wide significant associations in the FHIT and PLEK2 genes. One SNP in the intron of the FHIT gene showed a significant association in the combined analysis of discovery and replication samples of MDD and depressive symptoms samples, and it exceeded a genome-wide significance threshold.

The significant locus (rs9825823, chr3: 61,082,153) maps to the intronic region of the FHIT gene, a tumor suppressor protein implicated in several cancers (36). FHIT is expressed in multiple brain regions (amygdala, anterior cingulate cortex, caudate nucleus, prefrontal cortex, hippocampus, and hypothalamus; http://www.gtexportal.org/home/gene/FHIT). It plays an important role in oxidative stress and level of DNA damage (37), biological processes implicated in $\operatorname{MDD}(38,39)$.
FHIT is a circadian clock modifier gene (40) and has been related to daytime sleepiness (41), which may be salient to the etiology of depression.

In a GWAS of recurrent, early-onset MDD, three SNPs located in the FHIT gene were among the strongest associations in the overall and sex-stratified analyses (8), although none had genome-wide significance. Genetic variants located in FHIT have been reported in genetic studies of anxiety (42), autism (43), mental stress (44), comorbid depressive syndromes and alcohol dependence (45), citalopram-induced side effects (46), and a latent class analysis of MDD symptoms (7), but none has met genome-wide significance.

Several methodological aspects should be discussed. First, we evaluated the depression continuum by combining cases from clinical populations diagnosed with MDD and participants from the general population who had been assessed for depressive symptoms. Such an inclusive approach may increase heterogeneity of the phenotype especially because lifetime MDD was evaluated, whereas depressive symptoms indicate past weeks only. If anything, such an approach would cause an underestimation of the effects because less information on depressive symptoms was obtained. However, the advantages of a large sample can outweigh the disadvantages of a less precisely defined phenotype. This has been observed in the GWAS of educational attainment that was successfully used as a proxy for intelligence (47). Our additional replication

Table 1. Meta-analysis Results of the PGC MDD GWAMA and the CHARGE Depressive Symptoms GWAMA (Discovery)

\begin{tabular}{|c|c|c|c|c|c|c|c|c|c|}
\hline \multirow[b]{2}{*}{ SNP } & \multirow[b]{2}{*}{ Chr } & \multirow[b]{2}{*}{$\mathrm{BP}$} & \multirow[b]{2}{*}{ Closest Gene } & \multirow[b]{2}{*}{ Location } & \multirow[b]{2}{*}{ Allele } & \multirow[b]{2}{*}{ MAF } & \multicolumn{3}{|c|}{ Combined Meta-analysis $^{a}\left(N_{\text {total }}=70,017\right)$} \\
\hline & & & & & & & Direction & z Score & $p$ \\
\hline rs9825823 & 3 & $61,082,153$ & FHIT & intron & $\mathrm{T} / \mathrm{C}$ & 0.46 & ++ & 5.8 & $8.2 \times 10^{-9}$ \\
\hline rs9323497 & 14 & $67,873,128$ & PLEK2 & intron & $\mathrm{T} / \mathrm{C}$ & 0.05 & ++ & 5.5 & $3.3 \times 10^{-8}$ \\
\hline
\end{tabular}

Allele: minor/major on the + strand. Direction of effect: CHARGE ( $n=51,258$; continuous outcome analysis), PGC $(n=18,759 ;$ of which 9240 were MDD cases).

BP, base pair; CHARGE, Cohorts for Heart and Aging Research in Genomic Epidemiology; Chr, chromosome; GWAMA, genome-wide association meta-analysis; MAF, minor allele frequency (GRCh37/hg19); MDD, major depressive disorder; PGC, Psychiatric Genomics Consortium; SNP, single nucleotide polymorphism.

${ }^{a}$ This analysis was weighted for number of participants with clinically significant depressive symptoms in the CHARGE study $(n=5976)$ and number of cases in the PGC study $(n=9240)$. 
Table 2. Replication Analyses and Final Meta-analysis of Discovery and Replication Stages

\begin{tabular}{|c|c|c|c|c|c|c|c|c|c|c|c|c|}
\hline \multirow[b]{2}{*}{ SNP } & \multirow[b]{2}{*}{ Chr:BP } & \multirow[b]{2}{*}{ Allele } & \multicolumn{2}{|l|}{$\begin{array}{l}\text { PGC Replication } \\
(N=20,171)\end{array}$} & \multicolumn{2}{|c|}{$\begin{array}{l}\text { HRS Replication } \\
\qquad(N=8157) \\
\end{array}$} & \multicolumn{2}{|c|}{$\begin{array}{l}\text { Overall Replication } \\
(N=28,328) \\
\end{array}$} & \multicolumn{4}{|c|}{$\begin{array}{c}\text { Meta-analysis of Discovery and Two Replication } \\
\text { Samples }(N=98,345)\end{array}$} \\
\hline & & & Direct $^{a}$ & $p$ & Direct & $p$ & Direct $^{b}$ & $p$ & MAF & Direct $^{c}$ & z Score & $p$ \\
\hline rs9825823 & $3: 61,082,153$ & $\mathrm{~T} / \mathrm{C}$ & +++-++-+-++-++ & .04 & + & .20 & ++ & 0.02 & 0.46 & +++ & 6.10 & $1.0 \times 10^{-9}$ \\
\hline rs9323497 & $14: 67,873,128$ & $T / C$ & +-+--++++-++ & .80 & - & .80 & +- & 0.9 & 0.05 & -++ & 4.61 & $4.0 \times 10^{-6}$ \\
\hline
\end{tabular}

Allele: minor/major on the + strand.

BP, base pair; Chr, chromosome; Direct, direction; HRS, Health and Retirement Study; MAF, minor allele frequency (GRCh37/hg19); PGC, Psychiatric Genomics Consortium; SNP, single nucleotide polymorphism.

${ }^{a}$ Order of the studies: Cognitive Function and Mood Study (CoFaMS), Psychiatric Arm of the Population-based CoLaus (PsyCoLaus) Study, MDD2000-Edinburgh cases, GENPOD/NEWMEDS, Depression Genes and Networks (DGN), Genetics of Recurrent Early-Onset Depression Phase II (GenRED2), Harvard i2b2, Janssen, Max Planck Institute of Psychiatry (MPIP) Munich Antidepressant Response Signature (MARS) Study, OMNlex, Queensland Institute of Medical Research (QIMR)-COEX, RADIANT Irish cases, RADIANT U.S. cases, RADIANT Denmark cases, Roche Study, Study of Health in Pomerania (SHIP)-TREND, TwinGene.

${ }^{b}$ Order of the studies: PGC Replication, Health and Retirement Study.

${ }^{c}$ Order of the studies: PGC Replication, Health and Retirement Study, combined meta-analysis of discovery samples.

analysis showed that increasing the sample size yielded a stronger association of the top hit with the depression continuum. It is complex to calculate statistical power of the current analysis because quantitative and qualitative measures were combined. In the current study, a genetic association with the depression continuum may reflect an effect on broad depressive phenotypes but could also be accounted for by an association with low levels of general well-being (12\%-18\% heritability) that co-occur with depressive symptoms (48). Second, we used a $p$-value-based meta-analysis because effect estimates could not be directly evaluated in a straightforward manner. Third, the heterogeneity of the imputation methods used in the PGC and CHARGE discovery samples might reduce the statistical power. However, different imputation references did not change the results in the published PGC MDD study (7).

In conclusion, in this large GWAMA of a broad depression phenotype, we detected a locus associated with depression in clinical and general population samples. Our results suggest the importance of a broader depression phenotype to identify genetic variants underlying depression. Large samples with different depression phenotypes may also help to disentangle the genetic background of different forms of depression.

\section{ACKNOWLEDGMENTS AND DISCLOSURES}

We acknowledge the essential role of the CHARGE consortium in development and support of this manuscript. CHARGE members include the Netherlands' Rotterdam Study; the National Heart, Lung, and Blood Institute's Framingham Heart Study, Cardiovascular Health Study, and Atherosclerosis Risk in Communities Study; and the National Institute on Aging's (NIA) Iceland Age, Gene/Environment Susceptibility Study. Core funding for the PGC is from the U.S. National Institute of Mental Health (U01MH094421). The PGC was supported by the National Institute of Mental Health (NIMH R01 MH094421 and NIMH R01 MH094421). The Health and Retirement Study is supported by the NIA (NIA U01AG009740). The genotyping was funded separately by the NIA (RC2 AG036495 and RC4 AG039029). Our genotyping was conducted by the National Institutes of Health (NIH) Center for Inherited Disease Research at Johns Hopkins University. Genotyping quality control and final preparation of the data were performed by the Genetics Coordinating Center at the University of Washington. The CoLausIPsyCoLaus study was and is supported by research grants from GlaxoSmithKline, the Faculty of Biology and Medicine of Lausanne, and the Swiss National Science Foundation (3200B0-105993, 3200B0-118308, 33CSCO-122661, 33CS30-139468, and 33CS30-148401).
The University of Edinburgh is a charitable body, registered in Scotland, with registration number SC005336. UD was supported by grants of the German Research Foundation (Grant No. FOR 2107; DA1151/5-1) and Innovative Medizinische Forschung of the Medical Faculty of Muenster (Grant Nos. DA120903, DA111107, and DA211012). DJM is an NRS Research Fellow supported by the Chief Scientist Office. AMM is supported by the Wellcome Trust (104036/Z/14/Z), The Dr Mortimer and Theresa Sackler Foundation, and a Senior Clinical Fellowship (SCD/12). GENPOD was funded by the Medical Research Council (G0200243), and we acknowledge the other coinvestigators: Phil Cowen, David Nutt, Tim J. Peters, and Deborah Sharp. This study makes use of data generated by the Wellcome Trust Case-Control Consortium. A full list of the investigators who contributed to the generation of the data is available from http://www. wtccc.org.uk. Funding for the project was provided by the Wellcome Trust (076113, 085475, and 090355). NEWMEDS: The research leading to these results has received support from the Innovative Medicine Initiative Joint Undertaking (115008), of which resources are composed of European Union and European Federation of Pharmaceutical Industries and Associations inkind contributions and financial contributions from the European Union's Seventh Framework Programme (FP7/2007-2013). Funding for the Netherlands Study of Depression and Anxiety and Netherlands Twin Registry studies was obtained from the Netherlands Organization for Scientific Research (NWO), Center for Medical Systems Biology (NWO Genomics), Netherlands Bioinformatics Centre/BioAssist/RK(2008.024), Biobanking and Biomolecular Resources Research Infrastructure (BBMRI-NL, 184.021.007); VU University's Institute for Health and Care Research and Neuroscience Campus Amsterdam; the European Science Foundation (EU/QLRT-200101254); the European Community's Seventh Framework Programme (FP7/ 2007-2013); European Network of Genomic and Genetic Epidemiology (HEALTH-F4-2007-201413); the European Science Council (ERC Advanced, 230374); Rutgers University Cell and DNA Repository (NIMH U24 MH068457-06); the Avera Institute, Sioux Falls, South Dakota (USA); and the NIH (R01D0042157-01A and MH081802, Grand Opportunity grants 1RC2 MH089951 and 1RC2 MH089995). Part of the genotyping and analyses was funded by the Genetic Association Information Network of the Foundation for the National Institutes of Health. Computing was supported by BiG Grid, the Dutch e-Science Grid, which is financially supported by NWO. The RADIANT studies present independent research part funded by a joint grant from the Medical Research Council (U.K.) and GlaxoSmithKline (G0701420) and by financial support from the National Institute for Health Research (NIHR) Biomedical Research Centre for Mental Health at the South London and Maudsley NHS (National Health Service) Foundation Trust and King's College London. The views expressed are those of the authors and not necessarily those of the NHS, the NIHR, or the Department of Health. The Genome-based Therapeutic Drugs for Depression (GENDEP) project was funded by the European Commission Framework 6 grant (EC contract reference: LSHB-CT-2003-503428). Lundbeck provided nortriptyline and escitalopram for the GENDEP study. GlaxoSmithKline and the U.K. NIHR of the Department of Health contributed to 
the funding of the sample collection at the Institute of Psychiatry (London). The collection of samples and genotyping of the Danish control subjects was supported by grants from the Danish Strategic Research Council, the Stanley Research Foundation, and H. Lundbeck AVS, and we thank David M. Hougaard, Section of Neonatal Screening and Hormones, Statens Serum Institute, Copenhagen, Denmark; Preben Bo Mortensen, National Centre for Register-based Research, Aarhus University, Denmark; and the Lundbeck Foundation Initiative for Integrative Psychiatric Research, iPSYCH, Denmark. The Study of Health in Pomerania (SHIP)-TREND is part of the Community Medicine Research net of the University of Greifswald, Germany, which is funded by the Federal Ministry of Education and Research (01ZZ9603, 01ZZ0103, and 01ZZ0403), the Ministry of Cultural Affairs, and the Social Ministry of the Federal State of Mecklenburg-West Pomerania, and the network Greifswald Approach to Individualized Medicine (GANI_MED) is funded by the Federal Ministry of Education and Research (03IS2061A). Genome-wide genotyping in SHIP-TREND-0 was supported by the Federal Ministry of Education and Research (03ZIK012). This work was also funded by the German Research Foundation (GR 1912/5-1). The Cognitive Function and Mood Study is supported by the Faculty of Health Science, University of Adelaide, Adelaide, Australia. We are grateful to the Janssen study volunteers for participating in the clinical studies, participating clinical investigators, and the staff for enabling patient recruitment and blood sample collection.

ND had full access to all the data in the study and takes responsibility for the integrity of the data and the accuracy of the data analysis. ND and SW conducted and are responsible for the data analysis.

We thank the staff at Janssen Neuroscience for sample banking, DNA extraction, quality control, plating, and clinical data anonymization, and we thank the staff at Illumina for genotyping of the Janssen DNA samples.

The funding sources had no role in the design and conduct of the study; collection, management, analysis, and interpretation of the data; preparation, review, and approval of the manuscript; or decision to submit the manuscript for publication.

PFS is a consultant for Pfizer Inc. All other authors report no biomedical financial interests or potential conflicts of interest.

\section{ARTICLE INFORMATION}

From the Genetic Epidemiology Unit (AD, CMvD, NA), Department of Epidemiology (KH, AF, ND, HT), and Department of Psychiatry $(\mathrm{KH}, \mathrm{HT})$, Erasmus University Medical Center, Rotterdam; Unit of Genetic Epidemiology \& Bioinformatics (HS), Department of Epidemiology (ATA), and Department of Psychiatry (CAT), University Medical Center Groningen, University of Groningen, Groningen; and Department of Biological Psychology (EdG, DB, JJH, GW) and Department of Psychiatry, Neuroscience Campus Amsterdam and EMGO Institute of Health and Care Research (GvG, YM, BWP, JHS), VU University, Amsterdam, The Netherlands. Department of Psychiatry (ND), Dokuz Eylul University, Izmir, Turkey. Department of Psychiatry (PFS), Center for Psychiatric Genomics (PFS), and Department of Genetics (SW), Genomic Medicine, University of North Carolina at Chapel Hill, Chapel Hill, North Carolina; Department of Epidemiology (JAS, SLRK, EBW, WZ) and Institute for Social Research (JDF, EBW, DRW), University of Michigan, Ann Arbor, Michigan; Stanley Center for Psychiatric Research (ECD, SR, JWS), Program in Medical and Populational Genetics (TE), and Program in Medical and Population Genetics (PLDJ), The Broad Institute of Harvard and MIT; Division of Endocrinology (TE), Boston Children's Hospital, Cambridge; Analytic and Translational Genetics Unit (SR) and Center for Experimental Drugs and Diagnostics (RP), Massachusetts General Hospital and Harvard Medical School; Psychiatric and Neurodevelopmental Genetics Unit (ECD, JWS), Center for Human Genetic Research, Massachusetts General Hospital; Department of Medicine (JMM), Section of General Internal Medicine, Boston University School of Medicine, Boston; Department of Neurology (PLDG), Program in Translational NeuroPsychiatric Genomics, Brigham and Women's Hospital, Harvard Medical School; Department of Neurology (PLDJ), Department of Genetics (TE), and Department of Psychiatry (ECD, JWS), Harvard Medical School; Solid GT (JAQ), Boston; Department of Biostatistics (KLL), Boston University School of Public Health; and Boston University and National Heart, Lung, and Blood Institute's Framingham Heart Study (KLL, JMM),
Framingham, Massachusetts; Rush Alzheimer's Disease Center \& Department of Neurological Sciences (DAB, JY), Rush University Medical Center; and Department of Preventive Medicine (MCC), Northwestern University Feinberg School of Medicine, Chicago, Illinois; Department of Psychiatry (PM, ACH, MLP, JPR), Washington University, St. Louis, Missouri; Department of Medicine (THM Jr), University of Mississippi Medical Center, Jackson, Mississippi; Translational Gerontology Branch (LF, TT), Nationa Institute on Aging, Baltimore, Maryland; Brown Foundation Institute of Molecular Medicine (MF), University of Texas Health Science Center at Houston, Houston, Texas; Department of Psychiatry (SPH), Kaiser Permanente San Francisco Medical Center, San Francisco; and Department of Psychiatry and Behavioral Sciences (DFL), Stanford University, Stanford, California; Department of Epidemiology (KK), Mailman School of Public Health, Columbia University; College of Physicians and Surgeons (MMW), Columbia University and New York State Psychiatric Institute; and Division of Psychiatric Genomics (SMP), Department of Psychiatry, Icahn School of Medicine at Mount Sinai, New York, New York; Janssen Research \& Development LLC (QSL), New Brunswick, New Jersey; Charles E. Schmidt College of Medicine (MLP), Florida Atlantic University, Boca Raton, Florida; Department of Psychiatry (JBP), University of lowa Carver College of Medicine, lowa City, lowa; Division of Cancer Epidemiology and Genetics (JS), National Cancer Institute, National Institutes of Health, Bethesda, Maryland; Group Health (SS), Seattle, Washington. Department of Psychiatry (UD), University of Marburg, Marburg; Department of Psychiatry and Psychotherapy (SR), Charité, Campus Mitte, Berlin; Institute of Human Genetics (SC, MMN), University of Bonn, and Department of Genomics (SC, MMN), Life \& Brain Center, Bonn; Institute of Neuroscience and Medicine (SC), Research Centre Jülich, Jülich; Department of Psychiatry and Psychotherapy (UD), University of Münster, Münster; Department of Psychiatry and Psychotherapy (HJG), Helios Hospital Stralsund; Department of Psychiatry and Psychotherapy (HJG, SVdA), University Medicine Greifswald; German Center for Neurodegenerative Diseases (HJG, SVdA), Site Rostock/Greifswald; Interfaculty Institute for Genetics and Functional Genomics (GH), University of Greifswald; Institute for Community Medicine (AT), University Medicine Greifswald, Greifswald; Max Planck Institute of Psychiatry (SK, SL, BM-M); Institute of Psychiatric Phenomics and Genomics (TGS), Ludwig-Maximilians-University; Department of Psychosomatic Medicine and Psychotherapy $(\mathrm{K}-\mathrm{HL})$, Klinikum rechts der Isar, Technische Universität München; and Munich Cluster for Systems Neurology (BM-M), Munich; Institute of Epidemiology II (K-HL), Mental Health Research Unit, Helmholtz Zentrum München, German Research Center for Environmental Health, Neuherberg; Department of Genetic Epidemiology in Psychiatry (MRie), Central Institute of Mental Health, Medical Faculty Mannheim/Heidelberg University, Mannheim; and Department of Psychiatry and Psychotherapy (TGS), University of Göttingen, Göttingen, Germany. Discipline of Psychiatry (TA, ATA, BTB), School of Medicine, University of Adelaide, Adelaide, South Australia; Queensland Brain Institute (EMB), University of Queensland, St. Lucia, Queensland; QIMR Berghofer Medical Research Institute (SDG, NGM, GWM, DRN), Brisbane, and Institute of Health and Biomedical Innovation (DRN), Queensland University of Technology, Brisbane, Queensland; and Department of Psychiatry and Psychiatric Neuroscience (GCS), School of Medicine and Dentistry, James Cook University, Townsville, Queensland, Australia. Division of Psychiatry (DHRB, T-KC, DJM, AMM), University of Edinburgh, Edinburgh; MRC Social, Genetic \& Developmental Psychiatry Centre Centre (GB, CML, MRiv, RU), Institute of Psychiatry, Psychology \& Neuroscience, King's College London; and Division of Psychiatry (GL), University College London, London; University of Exeter Medical School (DJL), Exeter; University of Liverpool (BM-M), Institute of Translational Medicine, Liverpool; and MRC Centre for Neuropsychiatric Genetics and Genomics (MCO, KET), Institute of Psychological Medicine and Clinical Neurosciences, School of Medicine, Cardiff University, Cardiff, United Kingdom. Translational Neuropsychiatry Unit (HNB), Department of Clinical Medicine; Department of Biomedicine and Centre for Integrative Sequencing (ADB), Aarhus University; The Lundbeck Foundation Initiative for Integrative Psychiatric Research (HNB, ADB OM), Aarhus; and Research Department P (OM), Aarhus University Hospital, Risskov, Denmark. Department of Psychiatry (EC, MP), Lausanne University Hospital, and Institute of Social and Preventive Medicine (ZK), Centre Hospitalier Universitaire Vaudois, Lausanne; Division of Medical Genetics (SC), Department of Biomedicine, University of Basel; and Roche 
Pharmaceutical Research and Early Development (ED, CH, JAQ, DU), Neuroscience, Ophthalmology and Rare Diseases Discovery \& Translational Medicine Area, Roche Innovation Center Basel, F Hoffman-La Roche Ltd. Basel, Switzerland. Centre for Integrative Biology (ED), University of Trento, Trento, Italy. National Institute for Health and Welfare (JGE), Department of Chronic Disease Prevention; Department of General Practice and Primary Health Care (JGE), University of Helsinki; Unit of General Practice (JGE), Helsinki University Central Hospital; Folkhalsan Research Centre (JGE, JL); Institute of Behavioural Sciences (JL, KR), University of Helsinki, Helsinki; and Vasa Central Hospital (JGE), Vasa, Finland. Estonian Genome Center (TE, AM) and Institute of Molecular and Cell Biology (AM), University of Tartu, Tartu, Estonia. Department of Psychiatry (MG), Trinity Centre for Health Science, Dublin, Ireland. CIBERSAM-Universidad de Granada (MRiv) and Instituto de Investigación Biosanitaria ibs.GRANADA (MRiv), Hospitales Universitarios de Granada/Universidad de Granada, Granada, Spain. Dalhousie University (RU), Halifax, Nova Scotia, Canada. Department of Medical Epidemiology and Biostatistics (PFS), Karolinska Institutet, Sweden.

ND and SW contributed equally to this work. HT and PFS contributed equally to this work.

Address correspondence to Henning Tiemeier, M.D., Ph.D., Department of Epidemiology, Erasmus University Medical Centre, P.O. Box 2040, 3000 CA Rotterdam, The Netherlands; E-mail: h.tiemeier@erasmusmc.nl.

Received Aug 03, 2016; revised Nov 11, 2016; accepted Nov 22, 2016.

Supplementary material cited in this article is available online at http:// dx.doi.org/10.1016/j.biopsych.2016.11.013.

\section{REFERENCES}

1. Murray CJ, Vos T, Lozano R, Naghavi M, Flaxman AD, Michaud C, et al. (2012): Disability-adjusted life years (DALYs) for 291 diseases and injuries in 21 regions, 1990-2010: A systematic analysis for the Global Burden of Disease Study 2010. Lancet 380:2197-2223.

2. Sullivan PF, Neale MC, Kendler KS (2000): Genetic epidemiology of major depression: Review and meta-analysis. Am J Psychiatry 157:1552-1562.

3. Kohli MA, Lucae S, Saemann PG, Schmidt MV, Demirkan A, Hek K, et al. (2011): The neuronal transporter gene SLC6A15 confers risk to major depression. Neuron 70:252-265

4. Lewis CM, Ng MY, Butler AW, Cohen-Woods S, Uher R, Pirlo K, et al. (2010): Genome-wide association study of major recurrent depression in the U.K. population. Am J Psychiatry 167:949-957.

5. Muglia P, Tozzi F, Galwey NW, Francks C, Upmanyu R, Kong XQ, et al. (2010): Genome-wide association study of recurrent major depressive disorder in two European case-control cohorts. Mol Psychiatry 15:589-601

6. Rietschel M, Mattheisen M, Frank J, Treutlein J, Degenhardt F, Breuer R, et al. (2010): Genome-wide association-, replication-, and neuroimaging study implicates HOMER1 in the etiology of major depression. Biol Psychiatry 68:578-585.

7. Major Depressive Disorder Working Group of the Psychiatric GWAS Consortium, Ripke S, Wray NR, Lewis CM, Hamilton SP, Weissman $\mathrm{MM}$, et al. (2013): A mega-analysis of genome-wide association studies for major depressive disorder. Mol Psychiatry 18:497-511.

8. Shi J, Potash JB, Knowles JA, Weissman MM, Coryell W, Scheftner WA, et al. (2011): Genome-wide association study of recurrent earlyonset major depressive disorder. Mol Psychiatry 16:193-201.

9. Shyn SI, Shi J, Kraft JB, Potash JB, Knowles JA, Weissman MM, et al. (2011): Novel loci for major depression identified by genome-wide association study of Sequenced Treatment Alternatives to Relieve Depression and meta-analysis of three studies. Mol Psychiatry 16: 202-215.

10. Sullivan PF, de Geus EJ, Willemsen G, James MR, Smit JH, Zandbelt T, et al. (2009): Genome-wide association for major depressive disorder: A possible role for the presynaptic protein piccolo. Mol Psychiatry 14:359-375.

11. CONVERGE consortium (2015): Sparse whole-genome sequencing identifies two loci for major depressive disorder. Nature 523:588-591.
12. Jansson M, Gatz M, Berg S, Johansson B, Malmberg B, McClearn GE, et al. (2004): Gender differences in heritability of depressive symptoms in the elderly. Psychol Med 34:471-479.

13. Johnson W, McGue M, Gaist D, Vaupel JW, Christensen K (2002): Frequency and heritability of depression symptomatology in the second half of life: Evidence from Danish twins over 45. Psychol Med 32:1175-1185.

14. Hek K, Demirkan A, Lahti J, Terracciano A, Teumer A, Cornelis MC, et al. (2013): A genome-wide association study of depressive symptoms. Biol Psychiatry 73:667-678.

15. Wray NR, Pergadia ML, Blackwood DH, Penninx BW, Gordon SD, Nyholt DR, et al. (2012): Genome-wide association study of major depressive disorder: New results, meta-analysis, and lessons learned. Mol Psychiatry 17:36-48.

16. Levinson DF, Mostafavi S, Milaneschi Y, Rivera M, Ripke S, Wray NR, et al. (2014): Genetic studies of major depressive disorder: Why are there no genome-wide association study findings and what can we do about it? Biol Psychiatry 76:510-512.

17. Lobo DM, Agius M (2012): The mental illness spectrum. Psychiatria Danubina 24(suppl 1):S157-S160.

18. Cuijpers P, de Graaf R, van Dorsselaer S (2004): Minor depression: Risk profiles, functional disability, health care use, and risk of developing major depression. J Affect Disord 79:71-79.

19. Cuijpers P, Smit F (2004): Subthreshold depression as a risk indicator for major depressive disorder: A systematic review of prospective studies. Acta Psychiatrica Scandinavica 109:325-331.

20. Prisciandaro JJ, Roberts JE (2009): A comparison of the predictive abilities of dimensional and categorical models of unipolar depression in the National Comorbidity Survey. Psychol Med 39:1087-1096.

21. Lewinsohn PM, Klein DN, Durbin EC, Seeley JR, Rohde P (2003): Family study of subthreshold depressive symptoms: Risk factor for MDD? J Affect Disord 77:149-157.

22. Remick RA, Sadovnick AD, Lam RW, Zis AP, Yee IM (1996): Major depression, minor depression, and double depression: Are they distinct clinical entities? Am J Med Genet 67:347-353.

23. Kessler RC, Zhao S, Blazer DG, Swartz M (1997): Prevalence, correlates, and course of minor depression and major depression in the National Comorbidity Survey. J Affect Disord 45:19-30.

24. Chen LS, Eaton WW, Gallo JJ, Nestadt G, Crum RM (2000): Empirical examination of current depression categories in a population-based study: Symptoms, course, and risk factors. Am J Psychiatry 157: 573-580.

25. Cuijpers P, Vogelzangs N, Twisk J, Kleiboer A, Li J, Penninx BW (2013): Differential mortality rates in major and subthreshold depression: Meta-analysis of studies that measured both. $\mathrm{Br} \mathrm{J}$ Psychiatry 202:22-27.

26. Ayuso-Mateos JL, Nuevo R, Verdes E, Naidoo N, Chatterji S (2010): From depressive symptoms to depressive disorders: The relevance of thresholds. Br J Psychiatry 196:365-371.

27. Sakashita C, Slade T, Andrews G (2007): Empirical investigation of two assumptions in the diagnosis of DSM-IV major depressive episode. Aust N Z J Psychiatry 41:17-23.

28. de Graaf LE, Huibers MJ, Cuijpers P, Arntz A (2010): Minor and major depression in the general population: Does dysfunctional thinking play a role? Compr Psychiatry 51:266-274.

29. Backenstrass M, Frank A, Joest K, Hingmann S, Mundt C, Kronmuller KT (2006): A comparative study of nonspecific depressive symptoms and minor depression regarding functional impairment and associated characteristics in primary care. Compr Psychiatry 47:35-41.

30. Radloff LS (1977): The CES-D Scale: A self-report depression scale for research in the general population. Appl Psychol Meas 1:385.

31. Rakofsky JJ, Schettler PJ, Kinkead BL, Frank E, Judd LL, Kupfer DJ, et al. (2013): The prevalence and severity of depressive symptoms along the spectrum of unipolar depressive disorders: A post hoc analysis. J Clin Psychiatry 74:1084-1091.

32. Bulik-Sullivan BK, Loh PR, Finucane HK, Ripke S, Yang J, Schizophrenia Working Group of the Psychiatric Genomics Consortium, et al. (2015): LD score regression distinguishes 
confounding from polygenicity in genome-wide association studies. Nat Genet 47:291-295.

33. Willer CJ, Li Y, Abecasis GR (2010): METAL: Fast and efficient metaanalysis of genomewide association scans. Bioinformatics 26: 2190-2191.

34. Stouffer S, Suchman E, DeVinnery L, Star S, Williams R (1949): The American Soldier, vol. 1: Adjustment During Army Life. Princeton, NJ: Princeton University Press, 56.

35. Cross-Disorder Group of the Psychiatric Genomics Consortium, Lee SH, Ripke S, Neale BM, Faraone SV, Purcell SM, et al. (2013): Genetic relationship between five psychiatric disorders estimated from genome-wide SNPs. Nat Genet 45:984-994.

36. Wali A (2010): FHIT: Doubts are clear now. Scientific World J 10: 1142-1151.

37. Karras JR, Paisie CA, Huebner K (2014): Replicative stress and the FHIT gene: Roles in tumor suppression, genome stability and prevention of carcinogenesis. Cancers 6:1208-1219.

38. Jorgensen A, Krogh J, Miskowiak K, Bolwig TG, Kessing LV, Fink-Jensen A, et al. (2013): Systemic oxidatively generated DNA/ RNA damage in clinical depression: Associations to symptom severity and response to electroconvulsive therapy. J Affect Disord 149: 355-362.

39. Lopresti AL, Maker GL, Hood SD, Drummond PD (2014): A review of peripheral biomarkers in major depression: The potential of inflammatory and oxidative stress biomarkers. Prog Neuropsychopharmacol Biol Psychiatry 48:102-111.

40. Byrne EM, Heath AC, Madden PA, Pergadia ML, Hickie IB, Montgomery GW, et al. (2014): Testing the role of circadian genes in conferring risk for psychiatric disorders. Am J Med Genet B Neuropsychiatr Genet 165B:254-260.

41. Gottlieb DJ, O'Connor GT, Wilk JB (2007): Genome-wide association of sleep and circadian phenotypes. BMC Med Genet 8(suppl 1):S9.

42. Luciano M, Huffman JE, Arias-Vasquez A, Vinkhuyzen AA, Middeldorp CM, Giegling I, et al. (2012): Genome-wide association uncovers shared genetic effects among personality traits and mood states. Am J Med Genet B Neuropsychiatr Genet 159B:684-695.

43. Tsang KM, Croen LA, Torres AR, Kharrazi M, Delorenze GN, Windham GC, et al. (2013): A genome-wide survey of transgenerational genetic effects in autism. PLoS One 8:e76978.

44. Nikpay M, Seda O, Tremblay J, Petrovich M, Gaudet D, Kotchen TA, et al. (2012): Genetic mapping of habitual substance use, obesityrelated traits, responses to mental and physical stress, and heart rate and blood pressure measurements reveals shared genes that are overrepresented in the neural synapse. Hypertens Res 35:585-591.

45. Edwards AC, Aliev F, Bierut LJ, Bucholz KK, Edenberg H, Hesselbrock V, et al. (2012): Genome-wide association study of comorbid depressive syndrome and alcohol dependence. Psychiatr Genet 22:31-41.

46. Adkins DE, Clark SL, Aberg K, Hettema JM, Bukszar J, McClay JL, et al. (2012): Genome-wide pharmacogenomic study of citalopraminduced side effects in STAR*D. Transl Psychiatry 2:e129.

47. Rietveld CA, Medland SE, Derringer J, Yang J, Esko T, Martin NW, et al. (2013): GWAS of 126,559 individuals identifies genetic variants associated with educational attainment. Science 340:1467-1471.

48. Rietveld CA, Cesarini D, Benjamin DJ, Koellinger PD, De Neve JE, Tiemeier H, et al. (2013): Molecular genetics and subjective well-being. Proc Natl Acad Sci U S A 110:9692-9697. 